\title{
FRBR: Application of the Model to Textual Documents
}

\section{Edward O'Neill and Maja Žumer}

The FRBR (Functional Requirements for Bibliographic Records) model was a revolutionary development that presented a new view of the bibliographic universe. Although FRBR has been widely accepted and extensively studied, actual implementations have been limited. This can be partly attributed to: (1) the vague and controversial definitions of the group 1 entities - work, expression, manifestation, and item, (2) that various types of information resources pose different issues, and (3) the rapid digitization of information. The various definitions of information resources are identified and reviewed here. To simplify the modeling, this study is limited to a single type of information resource: textual documents consisting of a sequence of words that may include non-textual material in the form of tables, symbols, equations, and/or illustrations. The FRBR model is analyzed in the context of textual documents with particular emphasis on digital documents to better understand the group 1 entities. An overview of the problematic aspects of the FRBR model is discussed and possible solutions are proposed.

T t has been twenty years since the International Federation of Library Asso1 ciations and Institutions (IFLA) Section on Cataloguing approved the final report for the Functional Requirements for Bibliographic Records (FRBR). ${ }^{1}$ Since then, FRBR has been widely accepted, extensively studied, and significantly revised. In 2002, an FRBR Working Group was formed "to provide a focal point within IFLA for the ongoing support and development of the conceptual model and to encourage the use of FRBR as a reference model for the biblio-

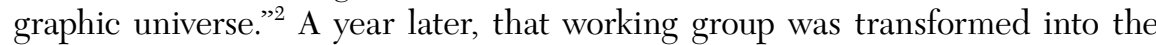
FRBR Review Group.

In 2005, the FRBR Review Group convened an invitational workshop to explore various aspects of the FRBR model. ${ }^{3}$ Following up on the problematic issues identified at the workshop, the FRBR Review Group established two working groups: the Working Group on the Expression Entity and the Working Group on Aggregates. FRBR was extended in 2008 to include name authority data (FRAD) and further extended in 2011 to encompass subject authority data (FRSAD). ${ }^{4}$ FRBR itself was revised in 2009 to incorporate updates to the model, including the changes proposed by the Working Group on the Expression Entity. ${ }^{5}$ The Working Group on Aggregates issued their report in 2011 (Aggregates Report) proposing how aggregates should be modeled. ${ }^{6}$ The IFLA Library Reference Model (LRM) represents the most recent development. LRM is a high-level conceptual reference model that consolidates the FRBR, FRAD, and FRSAD models. "LRM issues from, but is distinct from, the three previous models in the FR family of conceptual models, FRBR, FRAD, and FRSAD."” 


\section{Literature Review}

Despite all the work that has been done, considerable confusion about the model remains and some aspects are still controversial. Although the FRBR model includes three groups of entities and the relationships among them, it is the group 1 entities (work, expression, manifestation, and item) that have generated the most discussion, confusion, and controversy. Why is there still so much controversy and confusion? It is not due to the lack of scholarly interest-FRBR may be the most studied current aspect of information organization. At least six FRBR books plus numerous reports, theses, and papers have been written on the topic. ${ }^{8}$ The FRBR Bibliography identifies more than five hundred publications, even though it has not been updated since $2008 .{ }^{9}$

Because many of the publications have been based on different understandings of Work-Expression-ManifestationItem (WEMI) model, it has been difficult to apply the model consistently across different types of resources. The goal of this study is to clarify the model by applying it to textual documents, a relatively homogeneous type of library material. To achieve this, the most important sources dealing with concepts such as documents, texts, and textual documents are first analyzed. After refining the concept of textual documents, definitions of WEMI entities for textual documents are discussed and their conceptual boundaries identified. This analysis placed textual documents in the context of the bibliographic universe and identified several problematic aspects of the FRBR model.

RDA was heavily influenced by FRBR but does not strictly adhere to its concepts. Tillett stressed the close relationship between RDA and FRBR stating, "All of these features of FRBR are incorporated into the new cataloging code now being developed, called RDA: Resource Description and Access." ${ }^{\prime 10}$ While RDA differs from FRBR in several areas, the difference in the treatment of aggregates is especially notable. In describing the group 1 entries, RDA specifies that "Each of these terms [work, expression, manifestation, item], depending on what is being described, can refer to individual entities, aggregates, or components of these entities (e.g., the term work can refer to an individual work, an aggregate work, or a component of a work)."11 FRBR is much more restrictive and limits aggregates to manifestations. Because aggregates are relatively common, this distinction is particularly significant.

Understanding the group 1 entities is critical to applying FRBR because they constitute the model's foundation. Names of group 1 entities were introduced or redefined in the FRBR context. While it is tempting to equate the FRBR terms with their earlier usage, the equivalents are imprecise. As a conceptual model, FRBR provides broad definitions but often lacks sufficient details for implementation. Leaving the implementation details to the cataloging rules can be advantageous because it provides the flexibility necessary to make bibliographic distinctions based "on the anticipated needs of users." ${ }^{\text {21 }}$ While this flexibility is desirable, unambiguous definitions for the group 1 entities are necessary for the exchange and reuse of bibliographic data. Sharing bibliographic data in today's global environment is only practical if the data are created following consistent applications of accepted standards. If cataloging rules are based on differing or ambiguous definitions, sharing the resulting bibliographic data will be difficult. The lack of clear definitions contributes to confusion because discussion and examples are often based on varying definitions of the FRBR entities.

To explain, clarify, and identify problematic aspects of FRBR, this study focuses on textual documents and will:

- Review the various definitions of documents, texts, textual documents, and other similar terms;

- Discuss the WEMI model in the contexts of textual documents;

- Examine how digital resources should be modeled;

- Review the structure and development of bibliographic families; and

- Identify problematic aspects of the FRBR model.

Focusing on this single relatively homogeneous resource type greatly simplifies the terminology. FRBR's scope includes all bibliographic "entities described in library catalogues and national bibliographies." ${ }^{.13}$ In addition to print materials such as books and journals, there is also an extremely diverse set of resources that includes motion pictures, still images, musical scores, sound recordings, games, web resources, mixed media, data, artifacts, etc. Each resource type is associated with a specialized vocabulary. A discussion of the FRBR model often requires phrases such as "physical object (e.g., a copy of a one-volume monograph, a single audio cassette, etc.)."

While the terms "textual work," "document," "text," and "textual document" are commonly used to describe bibliographic entities and are often used interchangeably, they lack generally accepted and unambiguous definitions. The library literature includes numerous attempts to define or clarify the terminology, but definitions that worked for print resources cannot easily be extended to digital resources. Buckland uses multimedia as an example of the terminology problem. Multimedia, which "used to denote multiple, physically-different media, is now of renewed interest, because, ironically, the multiple media can be reduced to the mono-medium of electronically stored bits." ${ }^{14}$

Defining a document has never been easy and is even more difficult for digital resources. Svenonius defined documents as "information-bearing messages in recorded form" and stated that "a document may assume a variety of 
material embodiments: a copy of a book, a video, a sound recording, text or images on the Internet, or a one-of-akind work such as a manuscript or a painting." ${ }^{5}$ Definitions of documents range from a relatively narrow view that a document is a printed object intended to be read without mediation to a broader view that a document is any object that has the potential to inform. FRBR defines a work as "a distinct intellectual or artistic creation." ${ }^{, 16}$ In LRM, a work is defined as "the intellectual or artistic content of a distinct creation." ${ }^{.17}$ Because works are defined as intellectual or artistic creations and documents embody works, documents are also limited to man-made intellectual objects.

Buckland provides several different definitions of a document: ${ }^{18}$

- Any source of information, in material form, capable of being used for reference or study or as an authority. Examples: manuscripts, printed matter, illustrations, diagrams, museum specimens, etc. (International Institute for Intellectual Cooperation)

- Any physical or symbolic sign, preserved or recorded, intended to represent, to reconstruct, or to demonstrate a physical or conceptual phenomenon. (S. Briet)

- A document is the repository of an expressed thought. (Donker Duyvis)

- A document ... a record on a more or less flat surface. (Ranganathan)

- He also noted that Briet considered anything that is the "object of study" to be a document, citing an antelope in a zoo as an example.

In an earlier paper, Buckland examined "informationas-thing" and distinguished between knowledge and information. ${ }^{19} \mathrm{He}$ argued that knowledge is intangible, but information is tangible and must be recorded. He identified three types of information: data, documents, and objects. Documents were broadly defined to encompass images and sound. "Objects are collected, stored, retrieved, and examined as information, as a basis for becoming informed," and Buckland cited rocks, fossils, bones, and other artifacts as examples. ${ }^{20}$ He differentiated between documents and objects based on their dimensionality-documents are twodimensional, objects are three-dimensional.

Buckland recognized that relying on dimensionality is problematic because it results in a map being categorized as a document while a globe is an object. Applying FRBR's intellectual or artistic requirement, rather than dimensionality, solves this problem by considering three-dimensional man-made objects (globes, statues, models) to be documents rather than objects. Objects are limited to natural objects (antelopes, stones, specimens, etc.). By combining Svenonius's definition with the FRBR creativity requirement, documents can be defined as a recorded intellectual or artistic message. Books, journals, manuscripts, videos, sound recordings, photographs, paintings, sculptures, and monuments would all be considered documents.

Data, the third element in Buckland's information triad, generally consists of numeric or tabular information that is not intended to be read. Technology enables the collection of massive amounts of data (e.g., temperatures from across the globe, book circulation statistics, economic trends). Unlike documents, data are not primarily intended for human consumption without further processing and refinement

\section{Textual Documents}

When documents are broadly defined as recorded intellectual or artistic messages, they form a category that encompasses most information resources. Textual documents are a subset of documents, and common definitions of textual documents include:

- Any object that can be "read," whether this object is a work of literature, a street sign, an arrangement of buildings on a city block, or clothing styles. It is a coherent set of signs that transmits some kind of informative message; ${ }^{21}$

- The set of words that constituted writing; ${ }^{22}$ and

- [A collection of] certain words into a certain sequence. $^{23}$

A textual document is defined here as A document consisting of a sequence of words that may include nontextual material in the form of tables, symbols, equations, and/ or illustrations. Textual documents are language dependent and can take the form of either writing or recorded speech. They are intended to be read or heard. The most common examples are printed books and journals, but they also include spoken audio recordings and Braille. This view of textual documents and their place in the bibliographic universe is shown in figure 1. Each of the basic information types has a number of subtypes. While there are many other important document types (motion pictures, still images, musical scores, games, music, etc.), they are beyond the scope of this study.

Figure 2 shows the classic WEMI model using different shapes to represent each of the group 1 entities. The cloud shape is used for works, the oval for expressions, the hexagon for manifestations, and the rectangle for items. Using distinctive shapes makes the recognition of each entity type easier. The following discussion focuses on digital resources and ways in which they can be accommodated by the FRBR model. To better accommodate digital resources, the descriptions of group 1 entities will emphasize their 


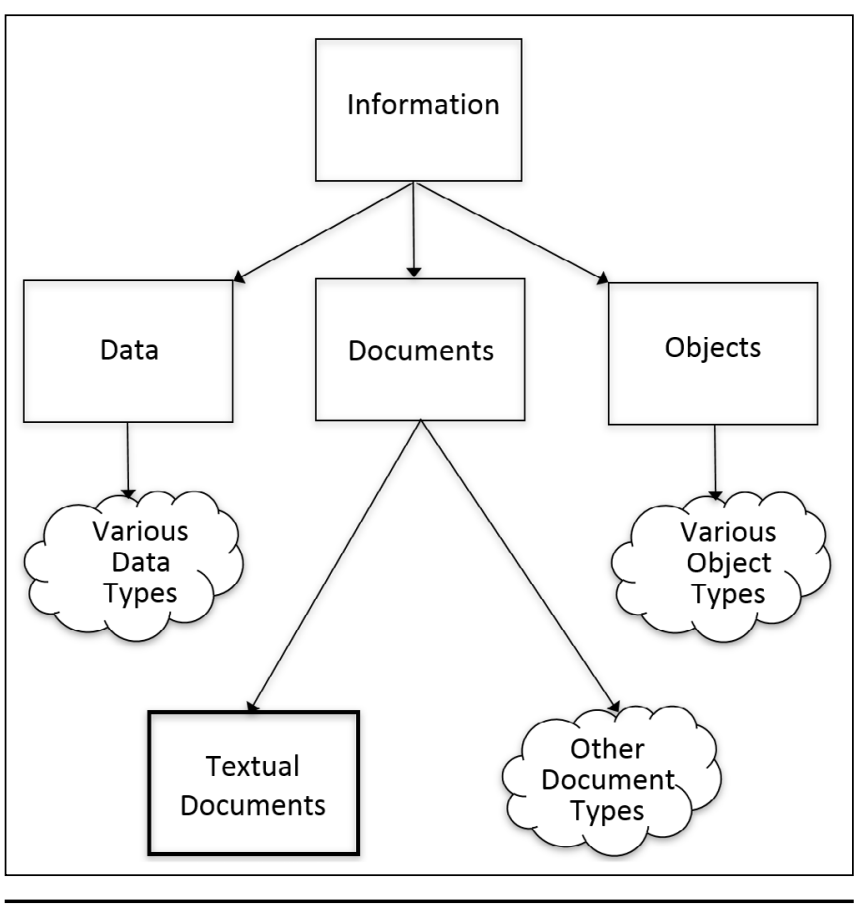

Figure 1. The Bibliographic Universe

function rather than their physical attributes or the media on which they are recorded.

Svenonius used the term "superwork" to represent the set of works derived from a common source. ${ }^{24}$ Although the term superwork is not used in FRBR, the concept can be represented with work-to-work relationships, provided that the derivation chain is known. A superwork encompasses all works (textual documents, motion pictures, music, etc.) derived from a common source. Smiraglia also discusses superworks, which he referred to as bibliographic families. He called the common source, "the first instantiation of a work," the progenitor. ${ }^{25}$ Smiraglia gave the progenitor special status, and identifying it is key to understanding and describing a bibliographic family.

The work family is a narrower concept than the bibliographic family; it is the set of group 1 entities associated with a single work. In a work family, the focus is on how a single work is realized in different expressions and, in turn, embodied in manifestations. Most works are simple and are realized in a single expression: a single manifestation that embodies the expression and the set of items exemplifying the manifestation.

Bennett, Lavoie, and O'Neill found that in OCLC's WorldCat, 94 percent of the works had a single expression and 78 percent had a single manifestation. ${ }^{26}$ Although their study was done prior to the revisions of definitions of expressions, their estimates are probably still reasonable. Even if only 6 percent of the work families contain multiple expressions, in absolute terms that is a very large number.

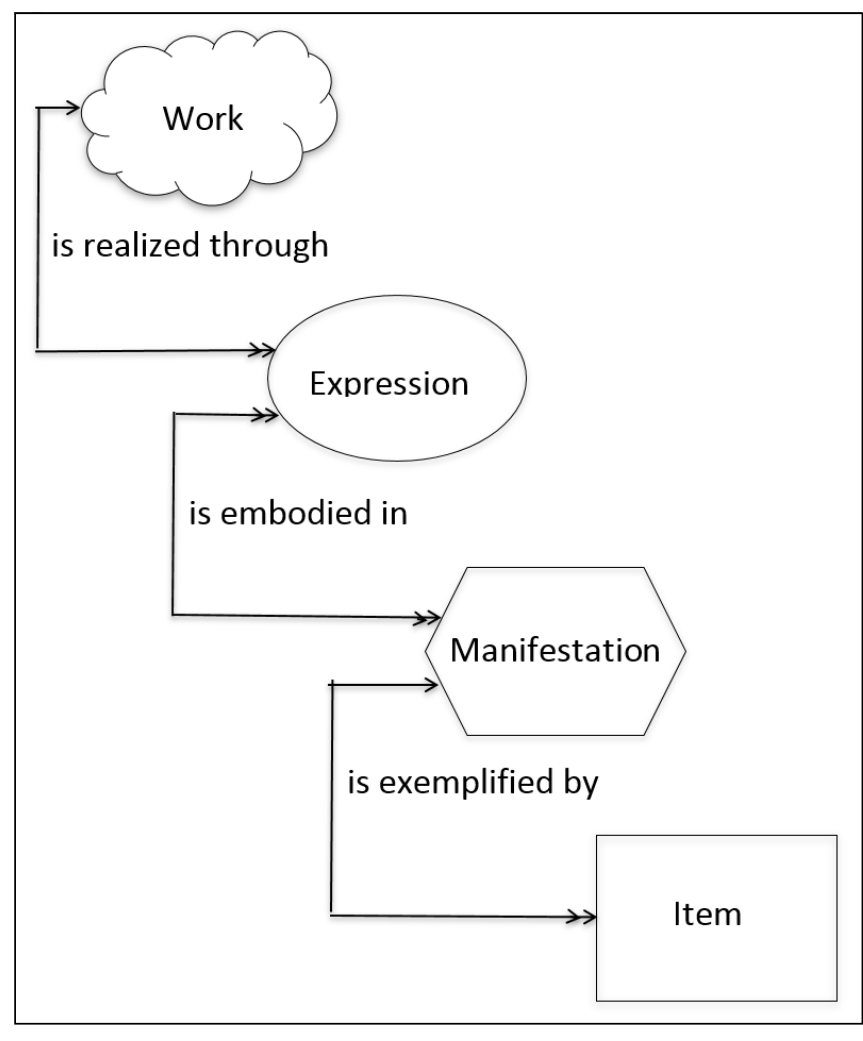

Figure 2. The WEMI Model

Since their study, WorldCat has grown to over 380 million bibliographic records, and there could be close to twenty million work families with multiple expressions in WorldCat. $^{27}$

These complex work families are generally important, highly held works. Some of these complex families have thousands of manifestations with millions of items. Understanding how these work families are formed and grow is essential to successfully applying the FRBR model. Work families typically start with a single expression with a single manifestation, the progenitor, and develop over time. The most common exceptions are multilingual publications that initially have expressions in different languages. The shift to digital has spurred the growth of new manifestations and expressions particularly for older, out-of-copyright works.

\section{Works}

In FRBR, "The work itself exists only in the commonality of content between and among the various expressions of the work." 28 The name associated with the progenitor usually serves as the name for the work and the entire set of associated documents. In most cases, documents derived from the progenitor are new expressions or manifestations of the work. FRBR acknowledges the difficulty of defining precise 
boundaries for works and states, "when the modification of a work involves a significant degree of independent intellectual or artistic effort, the result is viewed, for the purpose of this study, as a new work." ${ }^{\text {"29 }}$ Thus, paraphrases, rewritings, parodies, adaptations, abstracts, digests, and summaries are considered as new works. However, although new editions and translations also involve a significant degree of independent intellectual effort, they are not viewed as new works.

\section{Expressions}

An expression is defined as "the intellectual or artistic realization of a work in the form of alpha-numeric, musical, or choreographic notation, sound, image, object, movement, etc., or any combination of such forms." ${ }^{\text {"30 }}$ LRM states that it is "a distinct combination of signs conveying intellectual or artistic content." ${ }^{, 31}$ For textual documents, FRBR specifies that an expression encompasses the specific content in the form of words, sentences, and paragraphs. ${ }^{32}$ The expression boundaries exclude aspects of physical form (e.g., typeface and page layout) that are not integral to the intellectual or artistic realization of the work. Manifestations can embody multiple expressions, so the inclusion of nonintegral supplemental material such as illustrations, notes, and forewords embodied in the manifestation does not alter the primary expression. Such augmentations are considered separate expressions of their own separate work(s). FRBR further states, "If a text is revised or modified, the resulting expression is considered to be a new expression but minor changes, such as corrections of spelling and punctuation, etc., may be considered as variations within the same expression."33

While explicitly identifying some of the types of changes that constitute a new expression, as a conceptual model, FRBR only provides the general framework, leaving the details to the cataloging rules. Revised or updated expressions of the same work are considered new, as are translations from one language to another. Although FRBR is less specific, either the audio recording of a written document or the transcription of a speech also results in a new expressions by changing the form of the document.

\section{Revision}

When expressions are updated or revised, each revision is considered a new expression. When the original FRBR report was amended and corrected in 2009, the 2009 edition became a new expression. Nonfiction works are more frequently revised and updated than are works of fiction. A classic nonfiction work, Gray's Anatomy, was initially published in 1858 and is now in the forty-first edition. ${ }^{34}$ Each edition is a distinct expression. During their publishing history, expressions can undergo extensive changes, including content revisions, title changes, and authorship changes.

A Guide to the Library of Congress Classification is another example. Immroth was the original author of the work, and it was initially published in 1968. The second edition, also by Immroth, was published three years later with the same title. With the third and fourth editions, the authorship was transferred to Chan, and the title was changed to Immroth's Guide to the Library of Congress Classification. The fifth edition, also by Chan, reverted back to the original title A Guide to the Library of Congress Classification. Following Chan's death in 2014, Intner and Weihs assumed responsibility for the work, and the sixth edition was published with the slightly modified title of Guide to the Library of Congress Classification, with Chan, Intner, and Weihs as the authors. Although both the title and authors have changed multiple times over its fiftyyear history, it is a single work with six distinct expressions.

\section{Translation}

When expressions are translated, each translation becomes a new expression. The translation of the FRBR report from English to French was a new expression. When there are multiple translations in different languages, each translation becomes a distinct expression. There are at least two French translations of The Expedition of Humphry Clinker, the first by Giono and d'Iver, and another later translation by Kleiman-Lafon. Each of these translations is a distinct expression.

For fiction, the progenitor is typically the source for the translation. However, for nonfiction, the progenitor is less likely to be the source. When a work has multiple revised or updated editions, the latest original-language edition is likely to be the source for the translation. If the source expression is different, even translations by the same person into the same language will result in a different expression.

\section{Form}

Textual documents can be written or spoken. There is often an audio edition of best sellers and other popular works. Additionally, many audiobooks are available for the blind and visually impaired. Through its National Library Service for the Blind and Physically Handicapped, the Library of Congress "administers a free library program of Braille and audio materials circulated to eligible borrowers ..." Audible, an Amazon company, claims to be the world's largest library of audiobooks. Audible targets a broad audience of listeners with "Anywhere, anytime listening ... at home, in the car, at the gym." ${ }^{36}$ A recorded reading of a written document is a distinct expression. Typically, the print edition will be the progenitor, and the audio edition will be 
a reading. However, the progenitor sometimes will be an audio expression, particularly for the transcription of legal proceedings, speeches, addresses, lectures, etc.

Extensive changes to a textual document will result in a different document type (e.g., a motion picture, a play, or a video game) and generate a new work. When a book is adapted for a motion picture or a video game, the resulting product is a new work, since the resulting product is no longer a textual document. Translations and revisions are generally easy to recognize. Although possible, it is atypical for a textual document to be translated and revised in a single step.

\section{Manifestations}

In terms of intellectual content and physical form, a manifestation normally reflects the items it exemplifies. Variations may occur between items if those variations are the result of actions that occurred after the item(s) were published (e.g., defacement, margin notes, highlighting, rebinding, etc.). Changes in the manufacturing process resulting in minor variations between items (e.g., worn type, different paper, binding, etc.) do not create a new manifestation unless such changes were substantive or intentional. Items from different printings exemplify the same manifestation unless there are also significant changes to the content or physical form.

\section{As FRBR explains:}

The boundaries between one manifestation and another are drawn on the basis of both intellectual content and physical form. When the production process involves changes in physical form, the resulting product is considered a new manifestation. Changes in physical form include changes affecting display characteristics (e.g., a change in typeface, size of font, page layout, etc.), differences in physical medium (e.g., a change from paper to microfilm as the medium of conveyance) and changes in the container (e.g., a change from cassette to cartridge as the container for a tape). Where the production process involves a publisher, producer, distributor, etc., and there are changes signaled in the product that are related to publication, marketing, etc. (e.g., a change in publisher, repackaging, etc.), the resulting product may be considered a new manifestation. ${ }^{37}$

From the intended reader's perspective, an expression embodied in any manifestation will have the equivalent content. Manifestations can differ in media type (e.g., printed book, e-book, microform), in their encoding (e.g., Word, Portable Document Format (PDF), Hypertext Markup
Language (HTML)), in their layout/presentation (e.g., font, pagination, page size), and/or in the other expressions embodied in it, but by definition, the manifestation will contain the equivalent content. Significant changes to an expression's content will result in a new expression. A user may prefer a particular manifestation for its physical form (print, e-book, microform), but all manifestations embodying the expression will have equivalent content. The following sections identify and describe the changes that could signify a new manifestation.

\section{Media}

FRBR specifies that "changes in physical medium (e.g., a change from paper to microfilm as the medium of conveyance) and changes in the container (e.g., a change from cassette to cartridge as the container for a tape)" will result in a new manifestation. ${ }^{38}$ A wide variety of media have been used to embody expressions, including:

- Paper (paper, parchment, scrolls, etc.);

- Film (transparent media);

- Magnetic media (tape, disks, etc.);

- Electronic media (flash memory, computer RAM);

- Optical media (CDs, DVDs, etc.); and

- Cloud storage (non-specific online storage)

Paper and film have commonly been used to record analog documents, while electronic, optical media, and cloud storage have been used primarily for digital documents. Magnetic media has been widely used for both analog documents (audio tapes) and digital documents, both those born digitally and those created from analog.

Analog documents generally are tightly bound to the media embodying them. Digital documents may be tightly bound to their media (an audiobook on CD or Braille) or loosely bound (an e-book stored in the cloud). Tightly bound documents are distributed on their media, while loosely bound documents are distributed online. In the latter case, we rarely know or care about the particular media on which they were stored prior to being distributed. For online documents, it is appropriate to consider cloud storage to be a single media type regardless of its actual physical form.

\section{Encoding}

A document can be recorded in either an analog (print) or digital form. Print documents consist of a sequence of visual characters and symbols, traditionally in the form of ink on paper. Spoken documents are recorded as sound waves. For analog spoken recordings on magnetic tape, the sound waves are converted into a magnetic field of varying strength that is stored as magnetized areas. Recording on other media, 
such as vinyl records, follows a similar pattern. Regardless of the recording medium, all analog recordings are stored such that they can be converted to acoustic sound waves.

To record a spoken document digitally, the sound wave must first be digitized. The digital recording is stored as a series of numbers representing samples of the amplitude of the sound wave over time. The resulting digitized audio can be stored on any media compatible with digital data. Like analog recordings, digital audio must be converted back to an analog sound wave to be heard. While the concept of digitization is simple, a variety of different formats (.wav, .mp3, aac, .m4a, etc.) have been used to encode sound. These formats differ in the quality of the recording (fidelity), the size of digital image, and digital rights management (DRM). Each of the formats requires specific software to convert the digital file back to a sound wave. Although many devices (newer CD players, portable media players, computers, etc.) are compatible with multiple formats, none are compatible with all. Most older CD players are only compatible with the .wav format and are unable to play the newer formats.

Written documents can also be digitally encoded using a variety of different schemas. PDF, HTML, ePub, Mobi, and Word are widely used standards for textual documents. Word is commonly used to create documents, and some documents are distributed as Word files. Scholarly journals often use PDF and HTML, and PDF, ePub, and Mobi are widely used for e-books. Many e-books add DRM to control their use and prevent unlicensed distribution. The use of DRM further limits the compatibility of digital documents.

Braille materials, while digital, are a unique type of textual document intended for the blind and visually impaired. Braille is the only form of digital encoding that is intended to be read. It was developed in the early nineteenth century and is generally considered to be the first digital form of writing. ${ }^{39}$ Although Braille predates computers by more than a century, Braille encoding is similar to binary-coded decimal (BCD) schemas used in early computers that used six bits to encode a character. This limits the number of possible characters to sixty-four: twenty-six letters, ten digits, plus various special characters.

When identifying manifestation boundaries, each unique encoding format (including any DRM layers) is a distinct manifestation. A book in Braille is a different manifestation from the printed edition on which it was based. An expression encoded in PDF is a different manifestation from the Word document from which it was derived.

\section{Publisher}

When the publisher changes, a new manifestation is created even when there are no other apparent changes in the document.

\section{Appearance}

When the production process involves changes in a document's appearance, the result is a new manifestation. But the operative term-production process-for digital documents differs from that of print documents. The process for print documents includes fixing the final appearance and producing items that are identical in terms of font, page size, and layout. For digital documents, however, the production process does not include creating the final page images that will be rendered post-production to meet the needs of the reader and capability of the display. Generally, either the apparent font size or the page layout is fixed during production and the other is set when the document is rendered. PDFs and ePubs are examples of these two approaches to document rendering.

PDF "is a file format used to present documents in a manner independent of application software, hardware, and operating systems. Each PDF file encapsulates a complete description of a fixed-layout flat document, including the text, fonts, graphics, and other information needed to display it. A PDF file also captures the formatting of documents from a variety of applications. ${ }^{340}$ The image of a PDF document will have a consistent appearance regardless of the device used to display it. The size of the image and the apparent font size is device dependent. On phone sized screens, the apparent font size will be small, making reading a PDF document difficult.

The PDF mimics print in appearance by maintaining the typeface, page layout, and pagination across display devices. For selected applications (such as rendering journal articles), this works well and can produce an item that is virtually indistinguishable from its print equivalent. However, because the size of the image is scaled for the device, displaying a PDF on a small display can result in an image with an unreadable small font. Because the font size changes depending on the size of the display, a strict interpretation of FRBR would imply that simply changing the size of the display creates a new manifestation. However, considering variant displays as distinct manifestations is contrary to the functional view of manifestations and results in the inconsistent treatment of print and digital documents.

In contrast, ePub documents used with iBooks, Apple's e-book application, are designed so that the image can be optimized for the particular device: an iPad, iPhone, iPod, or Mac. "Read one page at a time, or turn your iPad on its side and view two pages at once. Read everything full screen, with no distractions, or read in white-on-black nighttime mode. Alter the look of most books by changing their text size and font." ${ }^{, 41}$ Instead of generating consistent images, iBooks adjusts the page layout to match the capabilities of the display and the reader's requirements. 
Figure 3 shows a page image on the left as it appears in print. The same text is shown in the upper right as a PDF document and in the lower right as an iBooks document as they would be rendered on an iPhone 7 . In the image of the PDF document, the layout is unchanged from the print but reduced in size to the point that it is almost unreadable. In the iBooks image, the layout is quite different and includes only a small portion of the text from the page image in a larger readable font. The image can be extensively modified by the reader; visually impaired readers can select a font size suitable for their needs. Documents can be rendered negatively in a white-on-black, a choice of fonts, different background page colors (white, sepia, gray, and black), or as a scroll.

The final appearance of digital documents is not fixed until they are displayed or printed, a process that occurs after the document has been distributed and over which the publisher has only limited control. Therefore, changes affecting the appearance that are made subsequent to its distribution should not be considered when determining whether the item is a new manifestation. It is assumed that allowing some changes in appearance is part of the publication/production plan and thus intrinsic to the manifestation and does not result in new manifestations.

\section{Aggregation}

The many-to-many relationship between expressions and manifestations in the FRBR model shown in figure 2 explicitly allows multiple expressions to be embodied in a single manifestation. When two or more independent expressions are published together in a single manifestation, an aggregate is formed. Modeling aggregates has been problematic because FRBR did not address them consistently or in sufficient detail. This lack of attention is somewhat surprising since aggregates are very common. O’Neill, Žumer, and Mixter estimated that over 20 percent of the items in library collections are aggregates. ${ }^{42}$

The Working Group on Aggregates was charged with analyzing and defining aggregates and proposing how they should be modeled. They defined an aggregate as "a manifestation embodying multiple distinct expressions" and identified three types of aggregates: ${ }^{43}$

- Collections are aggregates of expressions of works that are similar in form, genre, or type. Examples include selected and collected works, anthologies, journals.

- Augmentations are aggregates that are formed when an expression is published with nonintegral supplemental materials such as illustrations, forewords, introductions, biographical notes, etc., because such supplemental materials are considered to be distinct expressions of their own separate work(s).

- Parallels are aggregates of expressions of the same work. Common examples include multilingual editions of poetry, multilingual official publications, and user manuals.

The intellectual effort of creating an aggregate is a distinct intellectual effort that creates a special type of work called an aggregating work. While the expression of an aggregating work will always be embodied in an aggregate manifestation, it need not be specifically described unless it is considered significant. An aggregating work is distinct 
from and excludes the works aggregated. This approach, originally proposed by the Working Group on Aggregates, was later incorporated into LRM. Even after the report was accepted by the FRBR Review Group, there continues to be occasional examples of improper modeling, possibly due to vagueness and inconsistency of examples of aggregates in FRBR and lack of visibility of the Aggregates Report. ${ }^{44}$

\section{Items}

Although often neglected, items are very relevant. Almost everything known about manifestations, expressions, and works is inferred from observing items. In FRBR, an item is described as a tangible object. For analog documents, it can either be a single physical object (a single volume monograph, a spoken recording on single CD), or it can be comprised of multiple objects (a multiple-volume monograph or a spoken recording on several cassettes). Coyle points out that the item is neglected in most discussions of group 1 entities "possibly because it is also the most clear" but that clarity does not extend to digital items. ${ }^{45}$ The item is the only WEMI object that is human readable-it is what the reader "sees" or the listener "hears."

Digital documents, with the exception of Braille, require additional processing to become readable. There is no digital entity similar in both function and form to an analog item. LRM stresses that the publishing of digital documents is specific because the resulting items are not necessarily identical and "the production plan will involve aspects that are not fully specified as they are not under direct control of the producer," such as the specific device used to store or display the item. ${ }^{46}$ In LRM, some variations among items are allowed because they are considered to be exemplars of the same manifestation. Different investigators have investigated digital items and a number of criteria for identifying digital items have been suggested.

\section{Physical Objects}

Floyd and Renear may have been the first investigators to explore the concept of an item in the digital world. They argue that because an item is defined as a concrete entity, identifying an item stored online is problematic. They note, "In most scenarios of information use in the digital world there is no visible, discrete, concrete physical object comparable to a copy of a book." ${ }^{\text {"47 }}$ They proposed PMEs [patterned matter and energy] as one of two possible views of items in the digital world. Floyd and Renear argue that PMEs, the individual physical states of the relevant portions of the computing system, are an appropriate candidate to be an item.

However, they state that PMEs, although they are concrete, are not usually considered to be bibliographic objects because of their brief existence. Floyd and Renear note that "careful analysis of other common item-level attributes, such as item identifier, condition, etc., will also reveal that these are rarely applied, strictly speaking, to a PME, but rather to some related abstract object, perhaps corresponding manifestation or expression., ${ }^{3 \$}$ Reinforcing their concern, PMEs are not human readable and appear to represent an intermediate step in the information delivery process - the rough equivalent to printing plates or stencils.

\section{Files}

Floyd and Renear also propose files as an alternate to PMEs but question what constitutes a file. They state that "there are patterns of practice and discourse around the word 'file' but it is far from clear whether there is a single univocal concept behind them, much less what that concept is." ${ }^{39}$ They suggest several possibilities with their assessments:

- Files are abstract objects, a sequence of bits or characters. This is clear, but would make the rhetoric of creation, destruction, and location metaphorical and would blur the distinction between item and manifestation.

- Files are logical fictions. Logical fictions such as the "the average plumber," raises the question of what entities will be involved in their explication.

- Files are "freestanding" social objects, like a debt or a corporation, but the nature of such social objects is controversial.

Like PMEs, files are not readable-they are not the final product in the information delivery process. If files are items, what is the object that is actually delivered to the reader? Files, at least as the term is used in computing, do not necessarily correspond to what are typically considered to be documents. For some types of digital documents, such as PDFs, there may be a one-to-one correspondence between a document and file, while other types of documents may consist of multiple files and, in those cases, an individual file is not a complete document.

\section{Function}

In their attempt to identify digital items, Floyd and Renear focused on the physical characteristics based on FRBR's description of items as tangible or concrete objects. It is somewhat ironic that the Functional Requirements for Bibliographic Records focuses on the item's physical form, not its function. An item, at least for print documents, is the object that the reader actually uses: the book or journal.

What then is the functional equivalent to the item for digital documents? Because digital documents are not 
readable, they cannot be the final exemplar. To become readable, a digital document must be transformed into an analog image and either printed or displayed. A printed copy of a digital document may appear identical to its print equivalent. If the printed copy is an item, does it not follow that a printed image of the digital document is also an item? Is an image of the document displayed on a monitor also an item? The function of the displayed image is the same as that of the printed page, but it is difficult to argue that an image displayed on a computer monitor, tablet, smartphone, or other type of display is physical, tangible, or concrete.

While LRM does not specifically drop tangibility as a requirement, it recognizes that applying the definition developed for analog items to digital resources is problematic. FRBR defines an item as "a single exemplar of a manifestation." ${ }^{\circ 0}$ The definition itself does not require items to be tangible objects, but FRBR continues to state that "an item is a concrete entity." For For analog documents, associating items with physical objects is logical, but it fails for digital documents. If the requirement that items must be concrete physical objects is dropped, the original definition can be extended to both analog and digital documents.

Identifying an item by its function rather than by its physical properties ensures that analog and digital resources are treated consistently. For textual documents, an item would be the entity that is actually used by the reader or listener. This revision has little, if any, impact on analog documents while clarifying that an image displayed on a monitor or similar device is also an item.

\section{Conclusion}

Twenty years after the adoption of the FRBR model, there is still confusion, and some aspects remain controversial. This can be attributed to several causes:

- FRBR is vague and imprecise, leading to different interpretations.

- When the FRBR model was initially developed, the terminology was still very print oriented.

- The three different functional requirements models (FRBR, FRAD, and FRSAD) were developed over an extended period.

- Developments such as RDA and, more recently,
LRM, have reduced but not eliminated much of the confusion and ambiguity.

This study focused on textual documents, the most common type of library resources. Buckland's concept of documents was adopted to be compatible with FRBR, and a new approach to categorize information resources was proposed. After defining documents, the modeling of textual documents was analyzed. In principle, conceptual models provide the bibliographic framework by defining, in the context of entity-relationship formalism, the entities, attributes, and relationships. The line between the conceptual model and the cataloging rules is often fuzzy. Clear guidelines in the form of cataloging rules must also be developed to successfully implement FRBR. When examples are used to illustrate the conceptual model, they frequently reflect implicit implementation assumptions influenced by current cataloging rules and practice.

FRBR aims to satisfy two conflicting goals: providing sufficient flexibility to allow cataloging rules that satisfy local needs, expectations, and practice while establishing standards to encourage and support the exchange and reuse of bibliographic data. Sharing bibliographic data in today's global environment is only practical if the data are created according to consistent standards.

The criteria used to determine when expressions or manifestations should be considered distinct were explored. Particular focus was given to two particularly difficult issues: aggregates and digital publishing. For textual documents, aggregates can be successfully modeled following the recommendations in the Aggregates Report. Issues arising from digital publishing have been more difficult to resolve because there has been little agreement regarding how the concrete entity requirement should be applied. The proposed solution is to focus on function rather than physical form. Viewing the item as a human readable entity that can be read or listened to provides a consistent approach for both analog and digital resources.

The FRBR models propose a new paradigm and should be implemented as soon as possible. LRM, the consolidated model, is a big step forward. This analysis of textual documents in the FRBR contexts is another step, but the other types of documents (music, video, images, etc.) also need to identified and analyzed to better understand FRBR and guide its application.

\section{References}

1. IFLA Study Group on the Functional Requirements for Bibliographic Records, Functional Requirements for Bibliographic Records: Final Report (München: K.G. Saur, 1998);
Olivia M. A. Madison, "The Origins of the IFLA Study on Functional Requirements for Bibliographic Records," Cataloging \& Classification Quarterly 39, no. 3-4 (2005): 15. 
2. IFLA, "About the FRBR Review Group," accessed May 26, 2017, www.ifla.org/about-the-frbr-review-group.

3. FRBR in 21st Century Catalogues: An Invitational Workshop (May 2-4, 2005), "Program and Proceedings," accessed May 25, 2017, www.oclc.org/research/activities/frbr/frbr -workshop/program.html.

4. IFLA Working Group on Functional Requirements and Numbering of Authority Records (FRANAR), Functional Requirements for Authority Data: A Conceptual Model (München: K. G. Saur, 2009); IFLA Working Group on the Functional Requirements for Subject Authority Records (FRSAR). Marcia Lei Zeng, Maja Žumer, and Athena Salaba, eds., Functional Requirements for Subject Authority Data (FRSAD): A Conceptual Model (Berlin: De Gruyter Saur, 2011).

5. IFLA Study Group on the Functional Requirements for Bibliographic Records, Functional Requirements for Bibliographic Records: Final Report (Amended and Corrected, 2009), accessed May 25, 2017, www.ifla.org/files/assets /cataloguing/frbr/frbr_2008.pdf.

6. IFLA Working Group on Aggregates, Final Report of the Working Group on Aggregates, September 12, 2011, accessed May 25, 2017, www.ifla.org/files/assets/cataloguing /frbrrg/AggregatesFinalReport.pdf.

7. Pat Riva, Patrick Le Bœuf, and Maja Žumer, IFLA Library Reference Model, (IFLA, August 2017), accessed February 25, 2018, www.ifla.org/files/assets/cataloguing/frbr-lrm/ifla -lrm-august-2017_rev201712.pdf.

8. Patrick Le Boeuf, ed., Functional Requirements for Bibliographic Records (FRBR): Hype or Cure-All? (Binghamton, NY: Haworth Information Press, 2005); Robert L. Maxwell, FRBR: A Guide for the Perplexed (Chicago: American Library Association, 2007); Arlene G. Taylor, Understanding FRBR: What It Is and How It Will Affect Our Retrieval Tools (Westport, CT: Libraries Unlimited, 2007); Karen Coyle, FRBR, Before and After: A Look at Our Bibliographic Models (Chicago: ALA Editions, 2016); Richard P. Smiraglia, Pat Riva, and Maja Žumer, eds., The FRBR Family of Conceptual Models: Toward a Linked Bibliographic Future (London: Routledge, 2013); Yin Zhang and Athena Salaba, Implementing FRBR in Libraries: Key Issues and Future Directions (New York: Neal-Schuman Publishers, 2009).

9. FRBR Review Group, FRBR Bibliography, accessed March 27, 2017, www.ifla.org/node/881.

10. Barbara Tillett, "FRBR and RDA: Resource Description and Access," in Understanding FRBR: What It Is and How It Will Affect Our Retrieval Tools, ed. Arlene Taylor (Westport, CT): Libraries Unlimited, 2007), 88.

11. Joint Steering Committee for the Development of RDA, Resource Description and Access, accessed September 25, 2017, http://access.rdatoolkit.org (section 1.1.5).

12. Functional Requirements for Bibliographic Records (2009), 21. 13. Ibid., 7 .
14. Michael Buckland, "What Is a 'Digital Document'?" preprint of article published in Document Numérique (Paris) 2, no. 2 (1998): 1.

15. Elaine Svenonius, The Intellectual Foundation of Information Organization (Cambridge, MA: MIT Press, 2000), 34.

16. Functional Requirements for Bibliographic Records (2009), 17.

17. Riva, Le Boeuf, and Žumer, IFLA Library Reference Model, 22.

18. Michael K. Buckland, "What Is a 'Document'?," Journal of the American Society for Information Science 48, no. 9 (1997): 805-6.

19. Michael K. Buckland, "Information as Thing," Journal of the American Society for Information Science 42, no. 5 (1991): 351.

20. Ibid., 353.

21. Wikipedia, “Text (literary theory)," accessed May 26, 2017, https://en.wikipedia.org/wiki/Text_(literary_theory).

22. Richard P. Smiraglia, The Nature of "A Work": Implications for the Organization of Knowledge (Lanham, MD: Scarecrow Press, 2001), 3.

23. Patrick Wilson, Two Kinds of Power: An Essay on Bibliographic Control (Berkeley: University of California Press, 1968), 6.

24. Svenonious, The Intellectual Foundation of Information Organization, 38.

25. Smiraglia, The Nature of "A Work," 3.

26. Rick Bennett, Brian F. Lavoie, and Edward T. O’Neill. “The Concept of a Work in WorldCat: An Application of FRBR," Library Collections, Acquisitions \& Technical Services 27, no. 1 (2003): 51.

27. OCLC, "Inside WorldCat," accessed January 4, 2017, www .oclc.org/worldcat/inside-worldcat.en.html.

28. La Boeuf, Functional Requirements for Bibliographic Records, 17.

29. Ibid., 18.

30. Ibid., 19.

31. Riva, Le Boeuf, and Žumer, IFLA Library Reference Model, 22.

32. Functional Requirements for Bibliographic Records (2009), 20.

33. Ibid, 20.

34. Wikipedia, Gray's Anatomy, accessed January 1, 2017, en.wikipedia.org/wiki/Gray’s_Anatomy.

35. Library of Congress, "That All May Read . . . ," accessed January 27, 2017, www.loc.gov/nls/.

36. Audible, "What Is Audible," accessed August 3, 2017, www .audible.com.

37. Functional Requirements for Bibliographic Records (2009), 22.

38. Ibid., 22.

39. Wikipedia, "Braille," accessed January 1, 2017, https:// en.wikipedia.org/wiki/Braille.

40. Wikepedia, "Portable Document Format," accessed January 1, 2017, https://en.wikipedia.org/wiki/PDF. 
41. Apple, "iBooks," accessed January 1, 2017, www.apple.com /ibooks/.

42. Edward O’Neill, Maja Žumer, and Jeffrey Mixter, "FRBR Aggregates: Their Types and Frequency in Library Collections," Library Collections, Acquisitions \& Technical Services 59, no. 3 (2015).

43. La Boeuf, Final Report of the Working Group on Aggregates, 3, 6 .

44. Riva, Le Boeuf, and Žumer, FRBR Review Group, Report of Activities, 2011-2012, accessed June 24, 2017, www.ifla .org/files/assets/cataloguing/frbrrg/activities_2011-2012.pdf.
45. Coyle, FRBR, Before and After, 90.

46. IFLA Library Reference Model, 88.

47. Ingbert R. Floyd and Allen H. Renear, "What Exactly Is an Item in the Digital World?," Proceedings of the American Society for Information Science \& Technology, 2007, 2.

48. Ibid., 3.

49. Ibid.

50. Functional Requirements for Bibliographic Records (2009), 24.

51. Ibid., 24. 